\title{
DESAFIANDO LA ENSEÑANZA UNIVERSITARIA PARA LA FORMACIÓN EN EDUCACIÓN DE PÁRVULOS
}

\author{
Challenging didactics in students of an Early Childhood Education career
}

\author{
MARIBEL CALDERÓN SOTO \\ Escuela de Educación Inicial, Facultad de Educación, Centro de Investigación en Educación \\ Universidad Bernardo O'Higgins \\ maribelcalderons@gmail.com \\ ANDREA FARÍAS DÉLANO \\ Departamento de Gestión de Postgrado. Universidad de Santiago de Chile \\ andrea.farias.d@usach.cl
}

\section{RESUMEN}

En la última década la formación docente ha girado hacia una inserción temprana en espacios reales de trabajo con el fin de vincular el desarrollo de habilidades de pensamiento superior con las complejidades de los contextos educativos. Pese a todo, el cambio en las dinámicas de enseñanza-aprendizaje universitarias aún es lento y requiere de un proceso de transformación que permita aprovechar la presencia de estudiantes en diferentes espacios educativos. El objetivo de este estudio fue evaluar la implementación de un ajuste didáctico en un curso para Educadoras de Párvulos que utilizó los espacios de formación práctica como un eje articulador entre asignaturas teóricas y prácticas mediante la elaboración de un trabajo de diagnóstico e implementación de estrategias educativas en aulas reales. La implementación de este ajuste didáctico fue evaluada bajo enfoque cualitativo de alcance exploratorio, mediante estudio de casos, participando siete estudiantes y una docente. Se realizaron entrevistas personales analizadas mediante método de comparación constante junto con la evaluación de los materiales y actividades realizadas durante el curso. Los resultados indican una positiva evaluación de la pertinencia, secuencia de actividades, un aumento de la motivación en estas a medida que transcurría el curso y una valoración de la simulación como estrategia de transferencia para la realidad educativa. Sin embargo, también se describe la dificultad para elaborar una perspectiva dialógica de acción-reflexión para la resolución de problemas pedagógicos que ayuden a avanzar hacia una comprensión más compleja del escenario educativo de parte de las estudiantes. 
Palabras clave: Articulación curricular; estrategias de enseñanza-aprendizaje; formación de profesores; resolución de problemas.

\section{ABSTRACT}

Notwithstanding the teacher training education has evolved towards an early insertion into real-work spaces to allow the link between the development of higher-order thinking skills and the complexities of real educational contexts, the change in the teachinglearning dynamics is still slow and so require a makeover to allow them to take advantage of current under-degree students and their different learning contexts. The objective of this study was to assess the implementation of effective teaching-learning strategies in an underdegree programme for early-education students who were encouraged to use a new set of lesson design during their practicum hours, as an effective articulator axe between theory and practice, in real learning contexts. This intervention was assessed under a qualitative, exploratory approach, through the case studies of seven students and one teacher. Personal interviews were conducted and by using a comparison method, and their new class materials were revised, too. Results reveal a high level of relevance, sequencing of the activities, an increase in motivation as the programme unfolded, and a positive result from this simulation as an effective approach to the educational reality. On the other hand, there are still some difficulties to develop a dialogic perspective of action-reflection dynamic for the resolution of pedagogical issues, and so, to be able to move forward to a deeper comprehension of real educational contexts.

Keywords: Curriculum articulation; teaching and learning strategies; teacher training; problem solving.

\section{INTRODUCCIÓN}

La demanda por educación de calidad y su vinculación con la formación de profesores desafían las prácticas tradicionales en la formación universitaria. En el área de la educación inicial se ha potenciado una inserción temprana a los espacios reales de trabajo, la que busca promover una toma de perspectiva respecto a las complejidades del aula y una integración entre los saberes y avances disciplinares y pedagógicos que pueden ayudar a generar aprendizajes en niños y niñas, considerando el proceso como un fenómeno situado.

En esa línea, es clave revisar y reconsiderar no sólo los saberes pedagógicos y disciplinares necesarios de manejar por los y las profesionales, sino también las estrategias, metodologías y prácticas de enseñanza que los formadores de formadores actuales utilizan para generar

84 | INTEREDU № 3 VOL. II (DicIEMBRE 2020) PÁGS. 83-102. ISSN: 2735-6523 
oportunidades de aprendizaje. Especialmente, propendiendo la reflexión en torno a la articulación y coherencia entre los saberes o conocimientos propios de la profesión, que son trabajados en las instituciones formadoras y las singularidades a las que se enfrentan los y las estudiantes al ingresar a contextos de trabajo de aula en su formación práctica.

De acuerdo a esto, se generó un diseño micro-curricular en un curso lectivo de la malla de formación de educadoras de párvulos en vinculación con el curso de Taller de Práctica, que hiciera más explícita la relación entre el conocimiento pedagógico y su aporte a las prácticas cotidianas en aula. De este modo, se generan procesos de reflexión y búsqueda de prácticas pedagógicas que intencionen la articulación teórico-práctica y el desarrollo del pensamiento superior.

En este artículo se expone la experiencia de este trabajo, describiendo la fundamentación teórica del dispositivo de formación. Asimismo, revisando los principales resultados de la evaluación de este, desde la perspectiva de las estudiantes y una docente, quienes fueron participantes en la formación.

\section{APRENDER EN EL CONTEXTO UNIVERSITARIO ACTUAL: DESAFÍOS PARA LA FORMACIÓN DOCENTE}

El mayor acceso a la formación universitaria o superior es un fenómeno mundial que ha tenido consecuencias en los modos, estrategias y herramientas utilizados tradicionalmente en las universidades (Rodriguez, 2018). Se plantea que el desafío para los docentes se puede sintetizar en la necesidad de generar condiciones de enseñanza para que todos los estudiantes puedan aprender de manera compleja (Claxton, 2001). En el ámbito de la formación de educadores y educadoras, se traduce en generar constantes procesos activos-deliberativos (Rodrígues, 2013) que se relacionen directamente con las capacidades metacognitivas de los estudiantes (Lamas y Vargas, 2016).

La literatura describe de manera extensa las comprensiones actuales respecto de qué se trata el aprendizaje propiamente humano y su relación con la enseñanza. Comprensiones que confluyen y divergen en relevar diversas dimensiones y elementos. Sin embargo, un punto de convergencia entre aquellas sería la relación inextricable entre las dimensiones biológicas, sociales y culturales que promoverían el desarrollo de habilidades cognitivas complejas durante la ontogenia (Cole, 1998; Ferreres, China, y Abusamra, 2012; Woodward, Sommerville, Gerson, Henderson y Buresh, 2009).

Tal como en otros ámbitos educativos, estas transformaciones han llevado a preguntarse por las características que actualmente son necesarias para la formación universitaria. Esto implica discutir en torno a los dispositivos de instrucción más adecuados, a cómo gestionar el conocimiento y cuáles serían los métodos de enseñanza que pudieran potenciar el desarrollo de habilidades de reflexividad en los estudiantes que les permitan 
insertarse en la sociedad del conocimiento y responder a los requerimientos propios de la época (Tedesco, 2000). Todos estos cuestionamientos, característicos de un mundo en constante cambio, parecen también hacer remecer nociones a la base de las tradiciones educativas, como por ejemplo, qué es lo que se entiende por y qué se espera que sean las situaciones o experiencias de aprendizaje en la formación universitaria.

\section{VOLVER SOBRE LA NOCIÓN DE APRENDIZAJE EN LA EDUCACIÓN FORMAL}

Las conceptualizaciones respecto de qué es aprender son diversas, considerando el enfoque desde el cual se define. Cassasus (2008) afirma la existencia de cinco formas en las que se ha conceptualizado el aprendizaje, tomando en cuenta el paradigma desde el cual se define: como un proceso de incremento de informaciones, como memorización, como acción, como generación de sentido y como reinterpretación de la realidad. Señala que en las tres primeras hay una concepción claramente conductista del aprendizaje y en las dos últimas las perspectivas teóricas a la base son las cognitivistas, constructivistas y humanistas. Todas ellas serían observables en las aulas educativas.

De acuerdo a Díaz (2010), dichas concepciones actúan como filtro para los nuevos aprendizajes, ya que, mientras más temprano se incorporen más difícil resulta modificarlas, tendiendo a autoperpetuarse de acuerdo a la experiencia. Por ello, resulta fundamental generar acciones didácticas que promuevan nuevas experiencias bajo nuevas concepciones de aprendizaje.

Desde una perspectiva neopiagetana, el aprendizaje en contextos de formación de jóvenes y adultos es entendido como transformación de estructuras de conocimiento. Los conocimientos, habilidades o actitudes previas se activan y se convierten en el punto de partida (Bourgeois y Nizet, 1997). Por lo que el aprendizaje sería el resultado de una tensión entre la resistencia y el cambio para volver a un estado de equilibrio. Se considera el proceso de aprendizaje no como una mera asimilación de conceptos o destrezas, sino como apropiación de una forma distinta de pensar. Esta forma de pensar se ha señalado como un pensamiento dialéctico, que considera las dimensiones objetivas y subjetivas del estudiante en su formación, incluyendo procesos cognitivos y afectivos (Pekrun, 2006).

En la formación práctica se daría un escenario propicio para el desarrollo de habilidades que pongan en tensión los saberes previos y disciplinares obtenidos durante los cursos lectivos. En este espacio se daría un encuentro natural entre la tradición dogmática de la universidad y la realidad de un centro de práctica que enfrenta y desafía al estudiante con un sinnúmero de experiencias concretas que operan en una dirección a veces contraria a la academia (Zeichner, 2010). 
Es así que, este nuevo escenario educativo en la formación profesional implicaría no solo reconocer la historia educativa de cada participante y los saberes pedagógicos de la academia, sino también tensionarlos de forma tal que permita a los aprendices generar nuevos modos de comprender y actuar frente a la realidad.

\section{LA FORMACIÓN PRÁCTICA TEMPRANA EN EDUCACIÓN DE PÁRVULOS: OPORTUNIDADES Y DESAFÍOS}

Una buena formación profesional se vincula a una inmersión gradual y sostenida en ambientes auténticos de trabajo. Sin embargo, la experiencia de estudiantes de pedagogía indica que no siempre es percibido como un espacio dialógico entre el saber disciplinarpedagógico y la realidad concreta (Gaete, Gómez y Bascopé, 2016). Este escenario se presenta más bien como un terreno de tensión entre dos realidades que no siempre se resuelve desde una perspectiva de desarrollo o transformación positiva para el estudiante y profesional (Calderón, 2020; Rufinelli, 2014).

Considerando la instalación de un dispositivo de aprendizaje en contexto de práctica, la generación de un espacio desafiante y que permita el logro de los objetivos por parte del estudiante emerge como una condición necesaria, aunque no suficiente. Si bien, cierto desajuste parece necesario, deben brindarse oportunidades para que el o la estudiante participe activamente y se involucre con la actividad pedagógica.

Este escenario desafía al tutor/a universitario a guiar al estudiante que aprende hacia una práctica y un actuar que él o ella misma no ve, pero de la cual ha tenido experiencia y debiese poseer un amplio conocimiento pedagógico. Por otro lado, en los procesos actuales de trabajo de supervisión, el rol de mediador del proceso de aprendizaje lo toma un profesor guía, quien tiene la experiencia concreta pero que no necesariamente comparte los énfasis y sentidos atribuidos al proceso de formación en la práctica (Calderón, 2020; Coiduras, París, Torrelles y Carrera, 2014).

Uno de los elementos relevantes de la formación en la práctica consiste en la posibilidad reflexionar sobre esta y utilizar conceptualizaciones teóricas para explicar lo que se observa, lo que se ejecuta. Luego, en función de aquello, proponer estrategias de enseñanza que faciliten una experiencia promotora de aprendizaje en niños y niñas. Desde una perspectiva históricocultural se trataría de trabajar en la zona de desarrollo próximo de la educadora en formación, promoviendo la adquisición de habilidades que puedan apoyar su proceso de toma de decisiones pedagógicas en la práctica.

Considerando el contexto descrito, se desarrolló una propuesta de ajuste didáctico en un curso para educadoras de párvulos en formación. Esta propuesta se sustentaba en un modelo didáctico activo-situado (Medina y Salvador, 2002), donde se favoreció el rol activo del estudiante y se promovió el desarrollo de su autonomía y responsabilidad. Este modelo 
permite el encuentro entre los conocimientos teóricos o saberes pedagógicos y el trabajo de generación de experiencias de aula que vincula ambos campos de formación. Es el o la estudiante el único actor que transita simultáneamente entre estos dos contextos educativos que están llamados al diálogo y la integración, pero como se ha señalado, no siempre se experimenta como tal.

A continuación, se describe el fundamento teórico que sostiene las decisiones de organización del dispositivo implementado en esta experiencia pedagógica en educación superior.

\section{FUNDAMENTACIÓN TEÓRICA}

LA INTEGRACIÓN DE CONOCIMIENTOS SOBRE EL DESARROLLO INFANTIL: UNA PROPUESTA DE ARTICULACIÓN

La intervención a la programación del curso consideró una realidad contextual que se sostiene sobre la pregunta respecto a cómo se guía el proceso de observación, intervención y reflexión permanente en contextos de trabajo real de manera remota. Esta forma de enseñanza desafía lo que tradicionalmente se conoce como triángulo pedagógico.

Tal como lo plantea Houssaye (1988, citado en Ibañez (2007), el triángulo pedagógico es representado por esta figura geométrica, donde cada vértice representa a un actor del proceso: el profesor, el alumno y el saber, quienes establecen relaciones por y para el aprendizaje. La enseñanza sería la relación entre profesor-saber, la formación la relación entre profesor-estudiante, y el aprendizaje la relación entre alumno-saber. De esta forma, sería posible estudiar las relaciones entre los actores que dificulten o promuevan el diseño de los procesos de aprendizaje.

Por su parte, Ibañez (2007) propone un modelo alternativo que considera al estudiante, el discurso didáctico del profesor y el mundo real normado por el currículo. El aprendizaje sería comprendido como una modificación del desempeño individual ante situaciones concretas y mediado por el discurso didáctico intencionado desde el profesor a través de diversas interacciones.

Tomando elementos de ambos modelos y comprendiendo el proceso de aprendizaje de ciertas habilidades con una temporalidad que se extiende más allá de una unidad acotada, se plantea que el trabajo de integración teórico práctico para este curso particular entrega pistas respecto a cómo podemos comprender el proceso de formación con dos escenarios de aprendizaje para el estudiante, planteando situaciones que favorezcan la integración teóricopráctica, factor clave para un buen ejercicio profesional.

Tal como se presenta en la Figura 1, al centro del proceso se encuentra el estudiante con el saber en relación. Estudiante -en este caso educadora en formación- partícipe de una 88 | INTEREDU № 3 VOL. II (DiCIEMBRE 2020) PÁGS. 83-102. ISSN: 2735-6523 
comunidad educativa que enfrenta un contenido de aprendizaje con sus conocimientos, experiencias previas y con cierto nivel de disposición hacia la actividad de formación. La flecha indica la relación bidireccional entre ambos elementos. Por un lado, el o la estudiante no se enfrenta en un vacío de conocimientos o comprensiones, sino que ya posee una construcción de significado previo. Por otro lado, este saber tampoco se concibe como una materialidad concreta e inmutable, sino como una forma de acción o como una forma de conocer que permite la toma de decisiones (Ibañez, 2007). Para un educador, un saber se encuentra siempre en relación con una realidad concreta que puede ser transformada. Los conocimientos ayudarán a diseñar, actuar y evaluar en consecuencia una situación pedagógica para otros. El saber dinámico se convierte, entonces, en una habilidad que puede ser transferida y utilizada en distintos contextos.

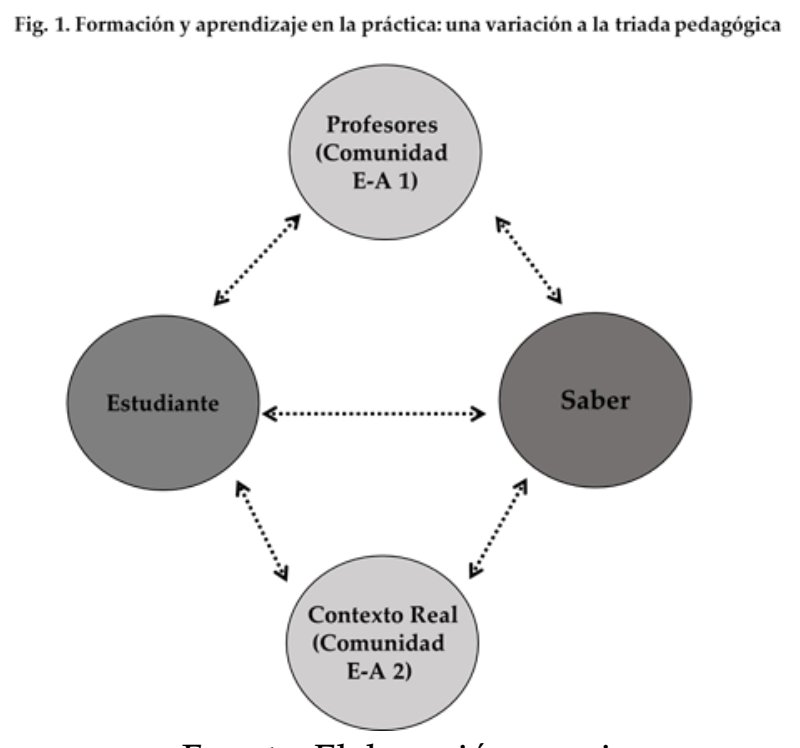

Fuente: Elaboración propia.

A un nivel de distancia de esta relación se encuentran los profesores de la universidad (comunidad de aprendizaje 1) y el contexto real (comunidad de enseñanza aprendizaje 2). Ambas comunidades tienen su propia naturaleza y sus propias prácticas culturales que movilizan el actuar de los estudiantes y se encuentran mediando en el proceso de enseñanza aprendizaje. El trabajo del profesor en la universidad y la actividad de los estudiantes se encuentran enmarcados en un curso donde el/la docente despliega herramientas y estrategias didácticas para que el estudiante se apropie de un saber disciplinar o pedagógico. En términos de Vigotsky, esta actividad se puede comprender de la siguiente forma: el docente estaría mediando el proceso de aprendizaje y trabajando en la zona de desarrollo próximo de los estudiantes, siendo las estrategias didácticas los mecanismos de andamiaje para el desarrollo de una habilidad específica (Baquero, 199; Labarrere, 2016). 
Por otro lado, la escuela -el contexto real- también es una comunidad de aprendizaje con sus propios ritmos y prácticas. El/la estudiante es un visitante que debe al mismo tiempo adaptarse y transformar en algún grado esa comunidad en la que participa durante un tiempo acotado.

Pero, ¿Qué hace que una tarea puede ser replicada en otro espacio de formación? Labarrere (2016) plantea que la zona de desarrollo próximo (ZDP), además de generar la adquisición de una competencia en el estudiante, también permite la construcción de sentido o móvil de la actividad. Este concepto permitiría comprender, que lo que ocurre en la sala de clase universitaria pueda llegar a ser valorado positivamente y transferido a otros espacios, cuando el estudiante le da sentido a la situación de enseñanza aprendizaje (E-A). Permite además mantener el dinamismo del proceso de E-A, pues en el contexto real donde participan estudiantes, profesores guías, prácticas y herramientas culturales, también pueden cuestionar ese sentido y el saber académico. Dicha situación devuelve la problemática al mundo académico en un ciclo virtuoso de reflexión.

\section{MÉTODO}

CONCRECIÓN DE UNA PROPUESTA CURRICULAR PARA LA INTEGRACIÓN DE CONOCIMIENTOS SOBRE EL DESARROLLO INFANTIL

La intervención se realizó entre marzo y agosto de 2017. Para la implementación de la propuesta curricular concreta, se consideró el objetivo de aprendizaje del curso Psicología del Aprendizaje: “Analizar el desarrollo integral de niños y niñas de educación parvularia, primero y segundo año de educación básica, mediante el uso de instrumentos estandarizados de evaluación que permiten tomar decisiones para mejorar los procesos de enseñanzaaprendizaje". Este objetivo se mantuvo sin variación, realizándose las modificaciones sólo a nivel de estrategias pedagógicas. Este objetivo se articuló con otro curso dictado en concurrencia: Taller de Práctica III. Durante el desarrollo de este curso-taller las estudiantes tienen la oportunidad de participar en aulas reales de trabajo, acompañadas por una educadora guía perteneciente al centro educativo. Las estudiantes participaron durante dos jornadas a la semana, reuniéndose con su supervisora en la universidad durante una clase de dos horas semanales. Las estudiantes generalmente realizaron en el establecimiento educativo labores de acompañamiento y planificación de experiencias de aprendizaje en un marco limitado de tiempo.

En la Tabla 1 se presenta una síntesis de la programación del curso, vinculando los objetivos a actividades en ambos espacios formativos. Se describe además la proporción de tiempo estimado en cada unidad, con un total de 14 semanas de intervención. 
Tabla 1. Objetivos específicos de aprendizaje del curso, contenido asociado y proporción del tiempo asociado a la unidad.

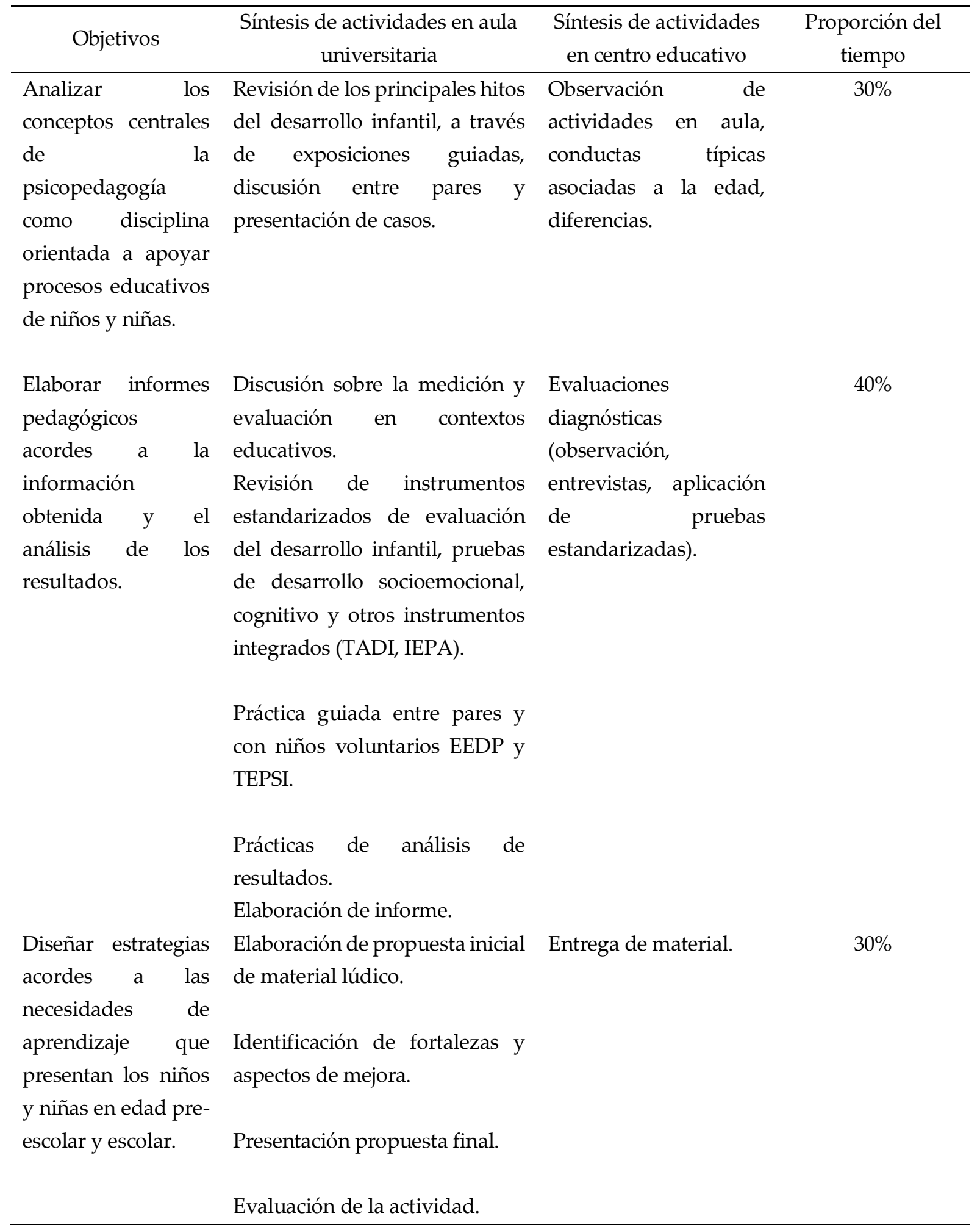

Fuente: Elaboración propia. 
Para organizar el trabajo en concurrencia se estableció contacto con la profesora del curso y con las educadoras guías de los establecimientos correspondientes, a fin de informar y solicitar la autorización a la institución y los consentimientos de los padres, madres y/o apoderados de los niños que participarían.

La programación del curso se dividió en tres momentos orientados por los objetivos instruccionales específicos. En cada uno de ellos se consideraron actividades en ambos espacios educativos. En un primero momento se trabajó en una profundización de conocimientos previos de las estudiantes respecto de diversos hitos del desarrollo infantil y mecanismos de promoción. La actividad se realizó mediante: exposiciones guiadas, discusión entre pares y presentación de casos. Todo ello debió dialogar con las observaciones realizadas por las estudiantes según el contexto de trabajo formativo que les correspondía participar para el taller de Práctica.

En un segundo momento, se discutió sobre el uso y utilidad de pruebas estandarizadas para evaluar el desarrollo infantil, presentando primeramente una descripción de pruebas usadas habitualmente en la evaluación de niños y niñas en Chile. Luego se profundizó sobre la utilización, aplicación y comprensión de dos pruebas estandarizadas que evalúan desarrollo y que son de uso frecuente en nuestro país. Estos son la Escala de Evaluación de Desarrollo Psicomotor EEDP para niños de 0 a 2 años (Rodríguez, Arancibia, y Undurraga, 1976), y el Test de Desarrollo Psicomotor TEPSI que se aplica en niños y niñas de 2 a 5 años (Heussler y Marchant, 2014). Se realizaron prácticas simuladas entre compañeras y también con niños y niñas visitantes, con previa autorización de los padres. Las estudiantes debían aplicar la prueba correspondiente al nivel donde se encontraban trabajando en su taller de Práctica III. Las aplicaciones de las pruebas se realizaron a tres niños y niñas en cada caso y esta actividad fue incorporada a la planificación de las actividades de práctica. En todas las aplicaciones se contó con una educadora a cargo del acompañamiento de la educadora en formación, con el fin de resguardar la seguridad y estabilidad de los niños y niñas.

La tercera etapa consistió en la elaboración de un informe y una propuesta de preparación de material lúdico-didáctico -elaboración de un prototipo- para la estimulación del desarrollo, según las necesidades detectadas en el levantamiento de información. En esta etapa también se consideró una evaluación final de la actividad de parte de las estudiantes y las docentes involucradas. Finalmente, el material fue construido en un formato más elaborado para entregar a las instituciones.

\section{OBJETIVO GENERAL}

Una vez implementada la propuesta diseñada, se propuso como objetivo evaluar el proceso de implementación de un cambio microcurricular en una asignatura teórica 92 | INTEREDU № 3 VOL. II (DicIEMBRE 2020) PÁGS. 83-102. ISSN: 2735-6523 
incorporando una articulación con un curso de taller de práctica en la formación de estudiantes de Pedagogía en Educación Parvularia en una universidad chilena.

Como objetivos específicos, se planteó primeramente evaluar la pertinencia de la propuesta de cambio microcurricular. En segundo lugar, se planteó establecer las fortalezas y debilidades de la experiencia, en término de los objetivos de aprendizaje.

\section{ENFOQUE, POBLACIÓN Y MUESTRA}

Se utilizó un enfoque de estudio de caso instrumental (Stake, 2010) en el sentido que el caso mismo si bien es importante, también lo es en relación al rol que tiene en la comprensión del fenómeno que interesa estudiar. Considerando este enfoque, las participantes de la experiencia fueron estudiantes de Educación de Párvulos de tercer año de una universidad privada chilena, quienes de manera concurrente se encontraban cursando la asignatura de Psicología del Aprendizaje y realizando el Taller de Práctica III. En total, fueron siete estudiantes y una docente quienes participaron de la implementación curricular, recogiéndose los datos desde tres de estas estudiantes y la docente.

\section{INSTRUMENTOS}

La recolección de información desde las estudiantes se realizó mediante una entrevista semiestructurada, para lo cual se generó un guión. El instrumento permitió recoger información respecto de los siguientes aspectos: línea de desarrollo de contenidos y actividades del curso, fortalezas y debilidades de los hitos más relevantes, percepciones respecto al proceso de involucramiento con las actividades y evaluación del material elaborado.

La perspectiva de la docente fue recogida por medio de un auto-reporte, elaborado mediante un registro escrito de las actividades.

Para la evaluación de las actividades se realizó una revisión crítica de la planificación y guías de trabajo en función de dos criterios: la pertinencia y la incorporación de cambios producto de las reflexiones emergentes que surgieron en las distintas etapas del proceso, sistematizando las oportunidades y problemas no previstos en la implementación de las actividades.

\section{PROCEDIMIENTO DE RECOGIDA Y ANÁLISIS DE DATOS}

El trabajo de indagación y evaluación del trabajo consideró el establecimiento de los resguardos éticos para los centros educativos y para las estudiantes a través la firma de consentimientos informados. 
Al ser un estudio de caso y una implementación didáctica se trató de indagar en dos aspectos fundamentales. Primeramente, la perspectiva de las estudiantes quienes experimentaron las acciones. Esta información se recogió por medio de las entrevistas, las cuales se realizaron tres meses después del cierre del curso. En segundo lugar, la perspectiva de la docente en tanto representa a la comunidad formadora y visibiliza las concepciones de lo educativo, presentes en el contexto de formación de profesores.

El procedimiento de análisis implicó dos procesos. En primer lugar, un análisis de contenido orientado a la búsqueda de contraste o comparación constante entre los relatos de experiencias de la docente y de las estudiantes. En segundo lugar, la reconstrucción de la secuencia de momentos de trabajo entre ambos campos de práctica. Ambos procesos fueron compartidos entre la docente y estudiantes con el fin de asegurar la veracidad de los datos, asegurando así la validación de los resultados.

\section{RESULTADOS}

Los resultados se presentan considerando dos aspectos. El primero consiste en una síntesis descriptiva de las distintas unidades trabajadas en cuanto a la pertinencia percibida por los participantes, entendiendo como pertinencia el grado en que los actores consideran que la unidad corresponde a la asignatura y que contribuye en algún sentido al propósito del curso. En ese marco, lo que se evalúa es la pertinencia de la estructura y organización del curso. Un segundo aspecto consiste en la descripción de las fortalezas y debilidades que emanaron desde el ejercicio de análisis de la evaluación de los participantes, donde lo que se evalúan son las tareas o acciones realizadas para promover oportunidades de aprendizaje con orientación al logro de los objetivos.

\section{PERTINENCIA DE LA ESTRUCTURA DE LA ASIGNATURA}

Para el objetivo didáctico 1 "Analizar los conceptos centrales de la psicopedagogía como disciplina orientada a apoyar procesos educativos de niños y niñas", las estudiantes evaluaron este proceso con una pertinencia media, no en relación a la atingencia de los contenidos, sino más bien a la sensación de repetición de estos.

"Al principio vimos cosas que ya nos han enseñado en otros ramos, eso podría haber sido más corto" (Estudiante 2).

Desde la evaluación que realiza la docente, ella consideró altamente pertinente el tiempo ocupado y las actividades realizadas para profundizar conceptos centrales del desarrollo infantil en distintas dimensiones.

94 | INTEREDU № 3 VOL. II (DiCIEMBRe 2020) PÁGS. 83-102. ISSN: 2735-6523 
"Si bien las estudiantes conocían algunos conceptos relevantes, se les hacía difícil seguir la relación entre el concepto y la realidad observada en el aula. Los conceptos se vuelven inútiles desde esa perspectiva" (Docente).

En cuanto al objetivo didáctico 2 "Elaborar informes pedagógicos acordes a la información obtenida y el análisis de los resultados", tanto la docente como las estudiantes consideraron que la temática fue adquiriendo relevancia a medida que se profundizaba en el uso de los instrumentos, la utilidad y la posibilidad de realizar en el contexto real una práctica ya simulada. Para la docente, la relevancia inicial estuvo puesta en la utilidad del instrumento y la información que de este emanó. Sin embargo, luego fue adquiriendo mayor relevancia en función del compromiso de las estudiantes con la actividad.

“Me sorprendí con lo que ocurrió con los instrumentos. Si bien al principio las estudiantes tenían una evaluación crítica de lo estandarizado por SIMCE, PSU y todo eso que se discute siempre, una vez que comenzamos con la aplicación de los instrumentos y la práctica, lo valoraron mucho. Fueron guiando la actividad, tanto así que propusieron trabajar con hijos de compañeras para que pudieran practicar la aplicación" (Docente).

Las estudiantes particularmente rescataron una serie de elementos contextuales respecto a la aplicación del instrumento -la situación de aplicación- que les permitió darse cuenta de sus habilidades y debilidades para el manejo de la conducta infantil y la búsqueda de estrategias para mantenerlos en la actividad.

"Lo de los test me sirvió mucho. Uno tiene más confianza al momento de aplicar el test, te 'craneas' (piensas estrategias) para que el niño mantenga la atención cuando aplicas el test" (Estudiante 2).

Además, perciben que para la comunidad educativa donde participaron también fue una actividad valorada.

"Al momento de empezar a aplicarlo encontré que se abren nuevas oportunidades. Yo encontré que en el jardín que yo estaba les habían aplicado tests a dos niños, pero no habían podido hacerlo con otros. Entonces que llegara una persona con conocimientos entre comillas a aplicar estos tests o de poder apoyar en ese sentido para ellos fue muy provechoso" (Estudiante 3).

Finalmente, en el tercer objetivo didáctico “Diseñar estrategias acordes a las necesidades de aprendizaje que presentan los niños y niñas en educación parvularia y básica", tuvo una alta valoración por parte de todas las participantes, aunque con matices de acuerdo al rol. Para la docente la actividad fue una integración de las acciones previas, que INTEREDU № 3 VoL. II (DicIEMBRE 2020) PÁGS. 83-102. ISSN: 2735-6523| 95 
demostrarían la capacidad de las estudiantes para transferir e integrar los distintos saberes en una propuesta acorde a las necesidades. Para las estudiantes implicó un proceso diferente al anterior, que se experimenta como una tarea colectiva desafiante que requiere tomar decisiones para alcanzarla.

“Al comienzo la aplicación era individual y luego nos reunimos con compañeras y tratamos de ver cuál era el área con más déficit. Revisamos bibliografía para evaluar el desarrollo y luego vimos qué era lo más factible de realizar rápido y no con tanto gasto" (Estudiante 1).

En la Tabla 2 se sistematizan los aspectos más relevantes de cada fase de evaluación que evidenciaron las estudiantes y la docente respecto de la estructura u organización del curso.

Tabla 2. Evaluación de la pertinencia percibida por docentes y estudiantes de las actividades de formación.

\begin{tabular}{|c|c|}
\hline Evaluación desde los docentes & Evaluación desde las estudiantes \\
\hline \multicolumn{2}{|c|}{ OBJETIVO DIDÁCTICO 1} \\
\hline Altamente pertinente. & Medianamente pertinente. \\
\hline $\begin{array}{l}\text { Conocimiento pedagógico como punto de } \\
\text { partida para la problematización del desarrollo } \\
\text { y su promoción en experiencias de aprendizaje. }\end{array}$ & $\begin{array}{l}\text { Proceso experimentado como extenso y repetitivo, } \\
\text { sensación de recurrencia de temas ya trabajados en } \\
\text { cursos anteriores. }\end{array}$ \\
\hline \multicolumn{2}{|c|}{ OBJETIVO DIDÁCTICO 2} \\
\hline Pertinencia creciente. & Pertinencia creciente. \\
\hline $\begin{array}{l}\text { Si bien era central en sus inicios, la práctica de } \\
\text { los instrumentos permitió mediar en los } \\
\text { procesos de reflexión de las estudiantes tanto }\end{array}$ & $\begin{array}{l}\text { Genera interés e incluso sorpresa reconocer las } \\
\text { diferencias entre niños frente a la evaluación a } \\
\text { través de instrumentos. }\end{array}$ \\
\hline $\begin{array}{l}\text { en la complejidad de la aplicación, la necesidad } \\
\text { de preparación y del valor informativo de los } \\
\text { instrumentos. }\end{array}$ & $\begin{array}{l}\text { El manejo de instrumentos estandarizados se } \\
\text { concibe como una habilidad que permite } \\
\text { enfrentarse de una forma más reflexiva en el espacio } \\
\text { educativo. }\end{array}$ \\
\hline \multicolumn{2}{|c|}{ OBJETIVO DIDÁCTICO 3} \\
\hline Altamente pertinente. & Altamente pertinente. \\
\hline $\begin{array}{l}\text { Proceso de cierre de la actividad de integración } \\
\text { entre los conceptos pedagógicos y su } \\
\text { concreción en el trabajo de aula. }\end{array}$ & $\begin{array}{l}\text { La actividad se convierte en desafiante en varios } \\
\text { sentidos. La necesidad de acordar colectivamente el } \\
\text { material adecuado, priorizar un aspecto del } \\
\text { desarrollo, realizar el material y considerarlo como } \\
\text { una evaluación sumativa hacia el final del semestre. }\end{array}$ \\
\hline
\end{tabular}

Fuente: Elaboración propia.

96 | INTEREDU № 3 VOL. II (DicIEMBRE 2020) PÁGS. 83-102. ISSN: 2735-6523 
Como síntesis de los resultados anteriores, se puede señalar que un primer elemento relevante de la evaluación realizada es la posibilidad de valorar las experiencias de los distintos actores de manera conjunta. Es decir, frente a los mismos objetivos y actividades, la percepción de los actores cambia en función del rol que le corresponde realizar. Respecto de este punto, se evidencia que la intencionalidad del docente que orienta la actividad no siempre es completamente visible y compartida por las estudiantes.

Otro aspecto que emerge es que las actividades relevantes para las estudiantes son aquellas que le permiten actuar de una manera más segura en el espacio de práctica. Las estudiantes valoraron significativamente el hecho de poder practicar en un espacio de formación protegido, para luego llevarlo a la experiencia real de evaluación en el espacio educativo correspondiente. Esta actuación aportaría a las prácticas habituales de las aulas y potenciaría un cierto sentido de competencia y autoeficacia respecto del valor de su trabajo en el establecimiento educativo y su utilidad futura.

Finalmente, la creación del material resultó pertinente y permitió la discusión y reflexión de algún aspecto específico de una dimensión del desarrollo, motivando a las estudiantes a ir más allá de las actividades rutinarias y promoviendo el pensamiento de orden superior. Igualmente, se debe consignar que esta producción final generó dificultades en su adecuación tanto por los tipos de materiales usados como en la especificación del mecanismo promotor de desarrollo que se proponía a través de este. Ello no impide que sea una actividad altamente valorada, como desencadenante de una actitud más activa frente al uso de materiales didácticos.

\section{FORTALEZAS Y ASPECTOS A MEJORAR PARA FUTURAS IMPLEMENTACIONES}

En la Tabla 3 se presenta una síntesis de aprendizajes de la experiencia didáctica, descrita como fortalezas y aspectos a mejorar. Entre las fortalezas se destaca la evaluación positiva de la trayectoria secuencial de las actividades, la participación activa e involucrada de las estudiantes en las prácticas en aula, la reflexión sobre la acción evaluativa y la complejidad de la evaluación del desarrollo que permite comprender de mejor forma el momento vital del niño/a y la posibilidad de promover experiencias de aprendizaje apropiadas. La secuencia de conocimientos pedagógicos hacia la aplicación de estos parece darle sentido a las actividades en los dos campos de acción donde participaron las estudiantes. Esto demuestra la importancia de generar los espacios en aula para que las educadoras en formación puedan describir, analizar y reflexionar sobre lo que observan. Contrastar las diferentes experiencias, vinculando esto a los conceptos, emerge como una oportunidad valorada positivamente. 
Tabla 3. Fortalezas y aspectos de mejora a partir de la experiencia de implementación del curso.

\begin{tabular}{|c|c|}
\hline Fortalezas & Aspectos a mejorar \\
\hline $\begin{array}{l}\text { Sensación de coherencia y de secuencia } \\
\text { natural en el trabajo recursivo de teoría y } \\
\text { práctica. }\end{array}$ & $\begin{array}{l}\text { Dificultad para comprender aspectos } \\
\text { interrelacionados del desarrollo y el aprendizaje. }\end{array}$ \\
\hline $\begin{array}{l}\text { Participación activa en procesos de práctica } \\
\text { guiada y simulada de parte de las } \\
\text { estudiantes. }\end{array}$ & $\begin{array}{l}\text { Los tiempos de trabajo universitario no } \\
\text { necesariamente dialogan con los tiempos de trabajo } \\
\text { en centro educativo, provocando desajustes en la } \\
\text { planificación. }\end{array}$ \\
\hline $\begin{array}{l}\text { Sensación de competencia o habilidad } \\
\text { desarrollada durante el curso por las } \\
\text { estudiantes. }\end{array}$ & $\begin{array}{l}\text { Dificultad para promover materiales concretos } \\
\text { apropiados a las necesidades detectadas en los } \\
\text { aprendices. }\end{array}$ \\
\hline $\begin{array}{l}\text { Desarrollo de la capacidad de reflexión en } \\
\text { torno a las condiciones y situaciones de } \\
\text { evaluación auténtica y guiada. }\end{array}$ & \\
\hline
\end{tabular}

Fuente: Elaboración propia.

En cuanto a los aspectos de mejora, un primer elemento se vincula al conocimiento y comprensión de aspectos relevantes del desarrollo. Se deben generar mecanismos para que las estudiantes perciban y comprendan los conceptos asociados al desarrollo como una profundización y no una repetición temática. Es decir, contar con programas de asignatura que reflejen dicha complejidad creciente en torno a una temática.

En segundo lugar y respecto a los tiempos de trabajo, la planificación académica requiere flexibilidad, debido a que las actividades planificadas en las sesiones de práctica deben estar articuladas con las solicitudes del curso Taller, con las necesidades educativas del establecimiento y las autorizaciones necesarias para la implementación del trabajo. Todo ello implica una alta coordinación entre los distintos actores en los diferentes contextos.

Finalmente y respecto del producto final -material didáctico-, si bien fue considerado pertinente como actividad, demoró más tiempo de lo estimado inicialmente. El hecho de ser una actividad calificada y realizada en la etapa final del semestre podría haber provocado cierta tensión o disminución del interés por la reflexión, lo cual merma la calidad de la entrega final.

\section{CONCLUSIONES}

El objetivo de este artículo fue presentar la evaluación de la implementación de una transformación didáctica orientada hacia la promoción de la integración teórico-práctica en un curso de formación básica en Pedagogía en Educación Parvularia. Como se ha venido 98 | INTEREDU № 3 VOL. II (DiCIEMBRE 2020) PÁGS. 83-102. ISSN: 2735-6523 
planteando de forma recurrente a lo largo de este escrito, una buena formación en educación implicaría considerar de manera temprana procesos de inmersión en espacios reales de ejercicio profesional. Sin embargo, esta inmersión implica un proceso de vinculación e integración en distintos niveles: curricular e interespacial formativo, que no siempre está acompasado en favor del aprendizaje de las y los estudiantes.

Los resultados indicaron una alta pertinencia de las actividades didácticas y su contribución al proceso formativo con algunos matices entre las percepciones de docente y estudiantes, valorándose positivamente este camino desde el campo más propio del conocimiento pedagógico hacia la intervención y creación para aportar a la solución de un problema real.

Las fortalezas y mejoras que se describen permiten establecer que es posible y necesario generar modificaciones curriculares que dialoguen entre espacios formativos. Este ejercicio de articulación curricular entre cursos de formación y cursos de práctica requiere una revisión y reformulación que debe ser emprendida por académicos y formadores, quienes orienten, intencionen y sean al mismo tiempo flexibles con el proceso, manteniendo siempre como norte los objetivos de aprendizajes o competencias esperadas a desarrollar.

Planteamos también que el modelo teórico de aprendizaje que organiza la relación entre el saber disciplinar y el saber de la experiencia concreta, es decir la relación entre teoría y la práctica, debe ser velada por los formadores de formadores, concebida no como una meta a alcanzar sino como un permanente ejercicio de contrastación que sostenga la relación dialéctica entre la reflexión y la acción, pero que permita la apropiación de saberes que mejoren el desempeño profesional.

Además de lo señalado anteriormente, es importante considerar las fortalezas y debilidades de esta experiencia ejecutada. Entre las fortalezas, se halla la propuesta de un criterio de evaluación de la estructura curricular de un curso, evidenciando las fortalezas y complejidades de la ejecución. Además de aportar a la discusión, considerando la oportunidad didáctica de vincular la experiencia concreta y real del proceso de formación en estudiantes de Educación Infantil con el conocimiento académico. Finalmente se plantea que el desarrollo de una percepción de propia competencia en las estudiantes dada la dinámica de las actividades, favorecería el proceso formativo y ampliaría su nivel de compromiso, validando el uso de estrategias didácticas de aprendizaje basado en problemas (ABP) o de simulaciones en las aulas universitarias.

Dentro de las limitaciones del estudio se considera la dificultad para generalizar los resultados, dado el acotado grupo de participantes. Sin embargo, la limitación mayor estuvo dada por la no incorporación de los profesores guías al proceso evaluativo, quienes fueron también partícipes activos del proceso de formación. Por otro lado, se plantea que para cerrar el proceso completo sería necesario considerar el uso de los materiales elaborados con las 
niñas y niños del contexto educativo. Con ello se puede profundizar la reflexión respecto de la complejidad del proceso de enseñanza aprendizaje y la toma de decisiones pedagógicas que se realizan en las aulas.

Finalmente, es importante señalar los aportes de este estudio para la comprensión de las condiciones de enseñanza aprendizaje en contextos de formación docente. Estos aportes describen y problematizan la experiencia concreta del proceso de formación en estudiantes de Educación Infantil, entendiendo además la vinculación entre ambos campos para el desarrollo de un pensamiento crítico y complejo de los y las profesionales de la educación.

\section{REFERENCIAS BIBLIOGRÁFICAS}

Baquero , R. (2012). Vigostky: sujeto y situación, claves de un programa psicológico. En J. Castorina, \& C. Mario, Desarrollo cognitivo y educación (págs. 61-86). Buenos Aires: Paidos.

Baquero, R. (1997). Vigotsky y el aprendizaje escolar. Buenos Aires: Aique.

Biggs, J. (2005). Calidad del Aprendizaje Universitario. Madrid: Narcea S.A. Ediciones.

Bourgeois, E., \& Nizet, J. (1997). Aprendizaje y Formación de Personas Adultas. París: Presses Universitaries de France.

Bruner, J. (2014). La educación, puerta de la cultura. Madrid: Machado Grupo de Distribución.

Calderón, M. (2020). Desafíos para la enseñanza y el aprendizaje en las prácticas progresivas en la formación de profesores. Una aproximación cualitativa en una universidad chilena. Profesorado. Revista de currículum y formación del profesorado. 24(2), 202-222.

Carretero, M. (2012). Cognición y Educación. En J. Castorina, \& M. Carretero, Desarrollo cognitivo y educación (págs. 97-112). Buenos Aires: Paidós.

Claxton, G. (2001). Aprender. El reto del aprendizaje continuo. Barcelona: Paidós.

Coiduras, J., París, G., Torrelles, C., \& Carrera, X. (2014). La evaluación de competencias en una experiencia de formación dual de maestros: diferencias y semejanzas entre tutores de escuela y universidad. Estudios Pedagógicos, XL(1), 29-48. doi:http://dx.doi.org/10.4067/S0718-07052014000200003

Cole, M. (1998). Cultural psychology:a once and future discipline. Cambridge, MA: Harvard University Press.

Contreras, D. (2009). Impacto de la educación preescolar sobre el logro educacional. Evidencia para Chile.

Cortázar, A., \& Vielma, C. (2017). Educación Parvularia Chilena: efectos por género y años de participación. Calidad en la Educación, 19-42. doi:https://doi.org/10.2307/1131396

100 | INTEREDU № 3 VOL. II (DiCIEMBRE 2020) PÁGs. 83-102. ISSN: 2735-6523 
Díaz, C. Martínez, P., Roa, I y Sanhueza, G. (2010). Los docentes de la sociedad actual: sus creencias y cogniciones pedagógicas respecto al proceso didáctico. Polis, 25, [En línea] URL: http://polis.revues.org/625

Ferreres, A., China, N., \& Abusamra, V. (2012). Cerebro, desarrollo y educación. En J. Castorina, \& M. Carretero, Desarrollo cognitivo y educación (I), 113-135. Buenos Aires: Paidós.

Gaete, A., Gómez, V., \& Bascopé, V. (2016). ¿Qué le piden los profesores a la formación inicial docente en Chile? En Centro de Políticas Públicas UC. Santiago.

García, C. (2007). La formación docente en la sociedad del conocimiento y la información: avances y temas pendientes. Olhar de Professor, 10(1), 63-90.

Heussler, I., \& Marchant, T. (2014). Test de Desarrollo Psicomotor2-5 años. Santiago: Universidad Católica de Chile.

Ibañez, C. (2007). Un análisis crítico del modelo del triángulo pedagógico. Una propuesta alternativa. Revista Mexicana de Investigación Educativa, 12(32), 435-456.

Kallio, E. (2011). Integrative thinking is the key: An evaluation of current research into the development of adult thinking. Theory \& Psychology, 21(6), 785-801.

Labarrere, F. (2016). La Zona de Desarrollo Próximo como eje del desarrollo de los estudiantes: de la ayuda a la colaboración. Summa Psicológica UST, 13(1), 45-56. doi:doi:10.18774/summa-vol13.num1-293

Lamas, P., Vargas-D’Uniam, J. (2016). Los niveles de reflexión en los portafolios de la Práctica Pre Profesional Docente. REDU. Revista de Docencia Universitaria, 14(2), 57-78.

Medina, A., \& Salvador, F. (2002). Didáctica General. Madrid: Pearson.

Moreno Vera, F., \& Agustín, D. (2017). Conocimientos disciplinares y pedagógicos de las educadoras de párvulos en Chile. Profesorado. Revista de currículum y formación del profesorado, 21(1), 233-261.

Pekrun, R. (2006). The Control-Value Theory of Achievement Emotions: Assumptions, Corollaries, and Implications for Educational Research and Practice. Educational Psychology Review, 18, 315-341. doi:https://doi.org/10.1007/s10648-006-9029-9

Rodrigues, R. (2013). El desarrollo de la práctica reflexiva sobre el quehacer docente, apoyada en el uso de un portafolio digital, en el marco de un programa de formación para académicos de la Universidad Centroamericana de Nicaragua (Tesis de Doctorado). Recuperado de http://hdl.handle.net/2445/43124

Rodriguez, S. (2018). La Universidad: una visión desde "fuera" orientada al futuro. 36(1), 15-38.

Rodríguez, S., Arancibia, V., \& Undurraga, C. (1976). Rodríguez S, Arancibia V, Undurraga C: Escala de evaluación de desarrollo psicomotor para niños entre 0 y 2 años. Santiago: Galdoc. 
Rufinelli, A. (2014). Dificultades de la iniciación docente: ¿iguales para todos? Estudios Pedagógicos, 50(1), 229-242. doi:http://dx.doi.org/10.4067/S0718-07052014000100014.

Sebastián, C., \& Lissi, M. (2016). El aprendizaje como proceso psicológico superior: hacia una comprensión histórico-cultural del desarrollo del proceso de aprender. En P. Freire, R. Moretti, \& F. Burrows, Aprender con otros. Aproximaciones psicosociales sobre el aprendizaje en contextos educativos (págs. 19-47). Santiago: Ediciones Universidad Alberto Hurtado.

Shulman, L. (1998). Theory, Practice, and the Education of Professionals. The Elementary School Journal, 98(8), 511-526.

Stake, R. (2010). Investigación con estudio de casos. Madrid: Morata.

Tedesco, J. (2000). Educar en la sociedad del conocimeinto. Buenos Aires. Fondo de Cultura Económica.

Woodward, A., Sommerville, J. A., Gerson, S., Henderson, A., \& Buresh, J. (2009). The Emergence of Intention Attribution in Infancy. Psychology of Learning and Motivation, $51,187-222$.

Zeichner, K. (2010). Rethinking the connections between campus courses and field experiences in college-and university-based teacher education. Journal of Teacher Education, 61(1-2), 89-99. doi:doi.org/10.1177/0022487109347671 